\title{
Magnetic torque evidence for the Fulde-Ferrell-Larkin-Ovchinnikov state in the layered organic superconductor $\kappa-(\mathrm{BEDT}-\mathrm{TTF})_{2} \mathrm{Cu}(\mathrm{NCS})_{2}$
}

\author{
B. Bergk, ${ }^{1}$ A. Demuer, ${ }^{2}$ I. Sheikin, ${ }^{2}$ Y. Wang, ${ }^{3}$ J. Wosnitza, ${ }^{1}$ Y. Nakazawa, ${ }^{4}$ and R. Lortz ${ }^{3, *}$ \\ ${ }^{1}$ Hochfeld-Magnetlabor Dresden (HLD), Forschungszentrum Dresden-Rossendorf, D-01314 Dresden, Germany \\ ${ }^{2}$ Grenoble High Magnetic Field Laboratory, CNRS, 25 avenue des Martyrs, Boîte Postale 166, F-38042 Grenoble \\ ${ }^{3}$ Department of Physics, The Hong Kong University of Science \& Technology, Clear Water Bay, Kowloon, Hong Kong \\ ${ }^{4}$ Department of Chemistry, Osaka University, 1-1, Machikaneyama, Toyonaka, Osaka, Japan
}

(Received 20 August 2010; published 14 February 2011)

\begin{abstract}
We present magnetic-torque measurements of the organic superconductor $\kappa$-(BEDT-TTF) $)_{2} \mathrm{Cu}(\mathrm{NCS})_{2}$ for inplane magnetic fields up to $32 \mathrm{~T}$. In this layered two-dimensional compound the superconductivity can persist even in fields above the Pauli limit of about $21 \mathrm{~T}$. There, a pronounced upturn of the upper-critical-field line occurs and the superconducting phase-transition line splits and forms an additional high-magnetic-field phase. $\kappa$-(BEDT-TTF $)_{2} \mathrm{Cu}(\mathrm{NCS})_{2}$ is a spin-singlet superconductor; therefore, such a superconducting high-field phase beyond the Pauli limit can originate only from Cooper pairing with finite center-of-mass momentum. The measurements are discussed in connection with a Fulde-Ferrell-Larkin-Ovchinnikov (FFLO) state, in accordance with earlier specific-heat observations. The torque experiments allow us to investigate the high-magnetic-field phase diagram and the FFLO state of $\kappa$-(BEDT-TTF $)_{2} \mathrm{Cu}(\mathrm{NCS})_{2}$ in great detail.
\end{abstract}

DOI: 10.1103/PhysRevB.83.064506

PACS number(s): 74.25.Dw, 74.70.Kn, 65.40.G-

\section{INTRODUCTION}

Type-II spin-singlet superconductors usually have two critical fields. Above $H_{\mathrm{c} 1}$, vortices penetrate the superconductor until, at the upper critical field $H_{\mathrm{c} 2}$ the current density of the screening currents around the vortex cores is beyond a critical value and the normal state is restored. Within this scenario, $H_{\mathrm{c} 2}$ is limited by orbital pair-breaking effects ${ }^{1}$ and at $H_{\text {orb }}\left(=H_{\mathrm{c} 2}\right)$ a continuous second-order phase transition is found. Layered two-dimensional materials exhibit cylindrical open Fermi surfaces, and, therefore, for in-plane magnetic fields, this orbital limit might be exceptionally high. Then eventually the Pauli paramagnetic limit for superconductivity becomes of importance. ${ }^{2,3}$ At the Pauli limiting field, the Zeeman-split Fermi surfaces no longer allow Cooper pairing with zero center-of-mass momentum. However, under certain circumstances the material might be able to remain superconducting above the Pauli limit by forming a novel superconducting state. A theory for such an unusual (the so-called FFLO) state was developed by Fulde and Ferrell, as well as Larkin and Ovchinnikov. ${ }^{4,5}$ Type-II superconductors that are able to approach the Pauli-limiting field $H_{\mathrm{P}}$ might increase their upper critical fields by "sacrificing" part of their volume to the normal state. Due to the Zeeman splitting of the Fermi surface, this can only be realized by Cooper pairing with a finite center-of-mass momentum. This leads to a spatial modulation of the superconducting order parameter above $H_{\mathrm{P}}$ with wavelength of the order of the coherence length. Consequently, superconductors with an FFLO state are supposed to exhibit another phase transition at the field $H_{\text {FFLO }}$ from the conventional superconducting into the FFLO superconducting state in the vicinity of $H_{\mathrm{P}}$ but below the enhanced $H_{\mathrm{c} 2}$.

For some years the high-field and low-temperature state of the heavy-fermion compound $\mathrm{CeCoIn}_{5}$ was discussed in the framework of the FFLO theory. ${ }^{6,7}$ The exact nature of the high-field state is, however, still the subject of controversy. ${ }^{8}$ Recently, neutron-scattering experiments revealed the existence of incommensurable antiferromagnetic order in this field-temperature region of what is now called the $Q$ phase. ${ }^{9}$ The two-dimensional organic superconductors, such as the nonmagnetic compound $\kappa$-(BEDT-TTF $)_{2} \mathrm{Cu}(\mathrm{NCS})_{2}$ (where BEDT-TTF is bisethylenedithio-tetrathiafulvalene) are further promising candidates to exhibit the FFLO state, ${ }^{10-13}$ since their high in-plane upper critical fields often exceed the Pauli limit. ${ }^{12-14}$ In several experiments on organic superconductors different features were interpreted as related to a development of the FFLO state in these materials: ${ }^{15-17}$ Early claims of the observation of an FFLO phase in $\kappa$-(BEDT$\mathrm{TTF})_{2} \mathrm{Cu}(\mathrm{NCS})_{2}$ were limited to rather broad features in the vortex stiffness. ${ }^{15}$ For $\lambda$-(BETS $)_{2} \mathrm{GaCl}_{4}$ and $\lambda$-(BETS) ${ }_{2} \mathrm{FeCl}_{4}$ (where BETS is bisethylenedithio-tetraselenafulvalene), kinks in the thermal conductivity ${ }^{16}$ and dip structures in the resistivity, ${ }^{17}$ respectively, suggested the existence of the FFLO state. Furthermore, some one-dimensional organic superconductors, such as (TMTSF) ${ }_{2} \mathrm{PF}_{6}$ (where TMTSF stands for tetramethyltetraselenafulvalene) show a characteristic upturn of their upper critical field for certain directions of the applied field. ${ }^{18}$ Nuclear magnetic resonance (NMR) experiments revealed evidence for a phase transition within the superconducting phase in (TMTSF) $)_{2} \mathrm{ClO}_{4} \cdot{ }^{19}$ However, thermodynamic proof for the FFLO transition was missing in these materials.

Previously, we reported clear thermodynamic evidence for the existence of an FFLO state in the organic superconductor $\kappa$-(BEDT-TTF $)_{2} \mathrm{Cu}(\mathrm{NCS})_{2}$ from specific-heat experiments. ${ }^{20}$ This was corroborated by preliminary magnetic-torque data ${ }^{21}$ for in-plane aligned magnetic fields. We have also confirmed that the compound fulfills all necessary prerequisites for an FFLO state, namely, that the samples are in the clean limit and exhibit a large Maki parameter of $\alpha=\sqrt{2} H_{\text {orb }} / H_{\mathrm{P}} \approx 8 \gg$ 1.8. The experiments revealed the characteristic upturn of the 
$H_{\mathrm{c} 2}$ line at $H_{\mathrm{P}}$, which was found to be in good agreement with theoretical predictions, ${ }^{20}$ and a thermodynamic phase transition within the superconducting phase.

An analogous phase diagram was acquired recently for the related two-dimensional superconductor $\beta^{\prime \prime}$-(BEDT$\mathrm{TTF})_{2} \mathrm{SF}_{5} \mathrm{CH}_{2} \mathrm{CF}_{2} \mathrm{SO}_{3}$ by use of radio-frequency penetrationdepth measurements. ${ }^{22}$ A pronounced upturn of $H_{\mathrm{c} 2}$ at low temperatures and indications for a second phase transition within the superconducting state close to the Pauli limit could also be determined. The high-field phase was interpreted as the FFLO state.

In this paper, we present magnetic-torque measurements on $\kappa$-(BEDT-TTF $)_{2} \mathrm{Cu}(\mathrm{NCS})_{2}$ in fields up to $32 \mathrm{~T}$ and temperatures down to $50 \mathrm{mK}$. Compared to our previous torque investigation, ${ }^{21}$ we have extended both the magnetic-field and temperature range to study the phase diagram in more detail. The large temperature and field range during the experiments, as well as the possibility to rotate the sample precisely with respect to the applied field, allowed us to investigate the highmagnetic-field phase diagram of $\kappa$-(BEDT-TTF $)_{2} \mathrm{Cu}(\mathrm{NCS})_{2}$ very thoroughly.

\section{EXPERIMENTAL TECHNIQUE}

$\kappa$-(BEDT-TTF $)_{2} \mathrm{Cu}(\mathrm{NCS})_{2}$ single crystals with masses of a few $100 \mu \mathrm{g}$ were grown by the standard electrochemicaloxidation method. Details of the method are illustrated in Ref. 23. The magnetic-torque measurements were carried out at low temperatures down to $50 \mathrm{mK}$ either in liquid ${ }^{4} \mathrm{He}$ or in the ${ }^{3} \mathrm{He}-{ }^{4} \mathrm{He}$ mixture of a dilution refrigerator and at high magnetic fields in a 32 as well as a $28 \mathrm{~T}$ resistive magnet at the Grenoble High Magnetic Field Facility by use of a capacitive-cantilever technique. The magnetic-torque signal of anisotropic samples is closely related to their bulk magnetization and, therefore, (if flux-pinning effects are negligible) a thermodynamic quantity. The cantilever sensor was placed on a low-temperature rotor which allowed us to align the sample with precision of about $0.01^{\circ}$ in the applied field. The detected de Haas-van Alphen (dHvA) oscillations in the normal state served as a quality test of the single crystals used in this study: In particular, at the lowest temperatures the dHvA oscillations display the typical sawtooth shape which points toward an exceptionally high sample quality. The investigated crystal showed the two characteristic dHvA frequencies in accordance with wellestablished literature data. ${ }^{24}$ For the main experiments the crystal was aligned with its superconducting BEDT-TTF layers (crystalline $b-c$ planes) parallel to the field. In order to find the proper parallel field alignment, the sample was rotated in small steps by minimizing the torque signal which is supposed to vanish for perfect parallel orientation.

Measurements were performed in the parallel orientation during field sweeps at fixed temperatures below the superconducting transition temperature (Sec. III). The first series of measurements was performed with the magnetic field aligned along the crystalline $b$ axis. Thereby, different crystals with identical phase diagram have been investigated. In a second series of measurements, we studied the in-plane angular dependence of the high-field magnetic phase diagram: The measurements were repeated but this time the magnetic field was applied parallel to the in-plane $b-c$ direction $\left(45^{\circ}\right.$ tilted from the first series of experiments). By this experiment we aimed at testing the influence of the pairing symmetry on the high-field phase diagram (Sec. IV). In a third series of experiments we finally examined what happens when the sample is turned in small steps out of the parallel orientation. This allowed us to gradually introduce orbital currents and to study how they compete with the FFLO state (Sec. V). The results of the experiments are discussed and summarized in Sec. VI.

\section{MAGNETIC-TORQUE EXPERIMENTS FOR MAGNETIC FIELDS APPLIED STRICTLY PARALLEL TO THE SUPERCONDUCTING LAYERS}

In Fig. 1 we present torque data at various fixed temperatures. At $8 \mathrm{~K}$, the transition at $H_{\mathrm{c} 2}$ is rather broad and the torque signal continuously approaches the normal-state zero value in the form of a broad kink. This is typical for spin-singlet type-II superconductors when orbital pair-breaking effects dominate the $H_{\mathrm{c} 2}$ transition. When the temperature is lowered toward $4 \mathrm{~K}$, the transition clearly sharpens similarly to our previous specific-heat data ${ }^{20}$ and transforms into a downward-step-like feature.

Such a feature in the magnetization points to a discontinuous first-order transition. As a further confirmation, a small hysteresis due to metastability could be resolved (as illustrated by the arrows in the data taken at $2.4 \mathrm{~K}$ and below). Apart from that, the main features in the data are reversible and do not depend on the field-sweep direction. The sharpening of the superconducting transition with decreasing temperature shows that Pauli paramagnetic effects become more and

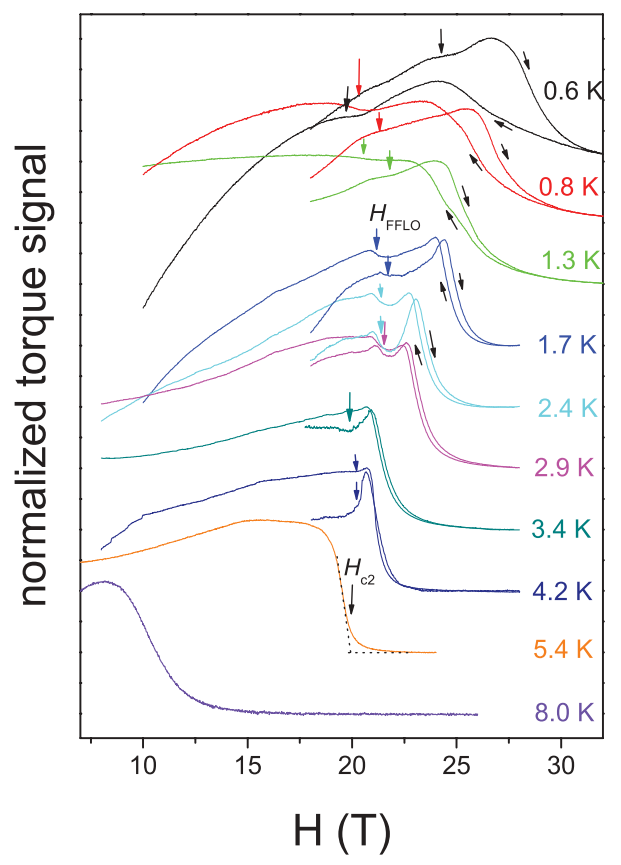

FIG. 1. (Color online) Magnetic-torque data of $\kappa$-(BEDT$\mathrm{TTF})_{2} \mathrm{Cu}(\mathrm{NCS})_{2}$ at various fixed temperatures for in-plane magnetic fields oriented along the crystalline $b$ direction. The direction of the field sweep is indicated by the arrows. Additional arrows mark the small diplike features, which we associate with the transition into the high-field FFLO state. 


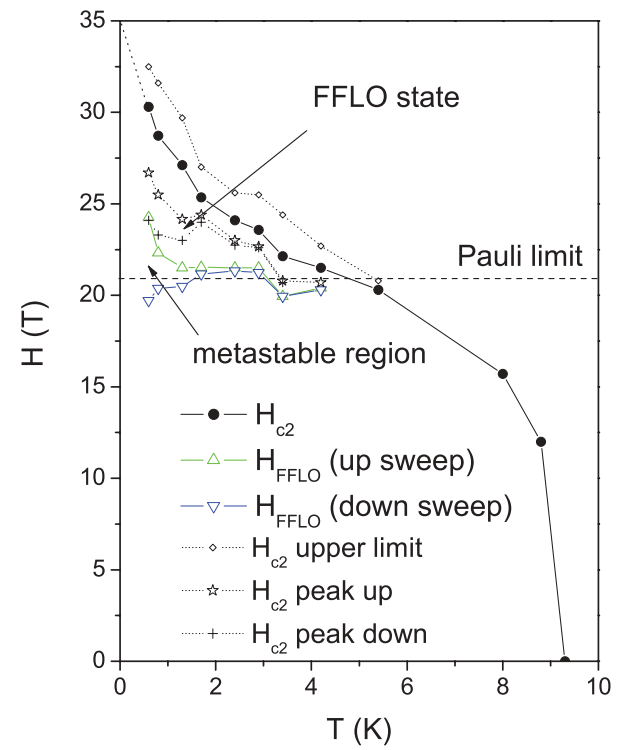

FIG. 2. (Color online) Magnetic phase diagram of $\kappa$-(BEDT$\mathrm{TTF})_{2} \mathrm{Cu}(\mathrm{NCS})_{2}$ for magnetic fields applied parallel to the superconducting layers ( $H$ is applied along the crystalline $b$ direction). The upper critical field $H_{\mathrm{c} 2}$ (solid dots, full line) and the transition between the Abrikosov state and the FFLO state $H_{\text {FFLO }}$ (triangles) are defined as indicated in Fig. 1. In order to illustrate the width of the transition we added three more criteria to define $H_{\mathrm{c} 2}$ : the maximum $H_{\mathrm{c} 2}$ value where the torque reaches approximately zero value $\left(H_{\mathrm{c} 2}\right.$ upper limit) and the fields where the maximum torque signal appears just at the lower onset of the transition upon field sweep up $\left(H_{\mathrm{c} 2}\right.$ peak up) and down ( $H_{\mathrm{c} 2}$ peak down).

more dominant in the pair-breaking mechanism around and beyond the Pauli limit at $H_{\mathrm{P}} \approx 21 \mathrm{~T} .^{20,21}$ Nevertheless, the superconducting state extends to even higher fields beyond this limit, where the transition into the normal state at $H_{\mathrm{c} 2}$ broadens again. Further, a small additional step (marked by arrows in Fig. 1) indicates a thermodynamic transition inside the superconducting state which always remains at fields close to $H_{\mathrm{P}}$. The small feature is very reversible and appears with both increasing and decreasing field. The downward step indicates that a fraction of the superconducting volume already turns normal well below $H_{\mathrm{c} 2}$. Such a decrease of the magnetic moment is expected at the transition into an FFLO state: The spatial modulation of the order parameter reduces the volume fraction of the superfluid density. ${ }^{25}$ Below $0.6 \mathrm{~K}$, pinning of magnetic flux manifests itself in the form of a larger hysteresis loop (data not shown and excluded from analysis, also because at the lowest temperatures the upper critical field exceeded our maximum field of $32 \mathrm{~T}$ ).

We use the midpoint of the small step to define $H_{\mathrm{FFLO}}$ and an extrapolation of the steepest slope of the torque signal to zero torque for $H_{\mathrm{c} 2}$. In this way we obtain the $H$-T phase diagram which is shown in Fig. 2 (note that the exact choice of criteria for the transition temperatures only marginally alters the phase diagram). The large initial slope of the $H_{\mathrm{c} 2}$ line at high temperatures is related to the strongly reduced orbital currents for in-plane field orientation. The rapid reduction of $T_{\mathrm{c}}$ once $H_{\mathrm{c} 2}$ exceeds about $12 \mathrm{~T}$ is related to the field-induced spin polarization. Above about $21 \mathrm{~T}, H_{\mathrm{c} 2}$ increases strongly toward lower temperatures, which indicates a modification in the superconducting condensate below $H_{\mathrm{c} 2}$. The phase diagram illustrates that the upturn can be associated with the additional phase transition within the superconducting state at $H_{\mathrm{FFLO}}$ since this transition approaches the $H_{\mathrm{c} 2}$ line at the onset of its upturn. Below $1 \mathrm{~K}, H_{\mathrm{c} 2}$ may be extrapolated to $35 \mathrm{~T}$ as the estimated upper critical field at zero temperature. Especially upon reducing the magnetic field, $H_{\mathrm{FFLO}}$ remains very close to the Pauli limit. Only at the lowest temperatures does the curve obtained from data measured upon increasing field show a minor upturn due to hysteresis effects related to metastability. This is very different from the phase diagram of $\mathrm{CeCoIn}_{5},{ }^{6}$ where the line of the $Q$ phase meets the $H_{\mathrm{c} 2}$ line only at the Pauli limit.

The phase diagram obtained by magnetic-torque measurements satisfactorily confirms the general behavior of our previous specific-heat data. Nevertheless, some obvious differences in the phase diagram also can be found. In contrast to the torque result, in the specific-heat data the second (FFLO) phase transition appears always close to $H_{\mathrm{c} 2}$. Unlike the field-dependent torque measurements the specific heat was obtained at various constant fields. In other words, the specific heat crosses the phase diagram along the temperature axis but the magnetization measurement along the field axis. Therefore, anomalies emerging at nearby fields that can be easily resolved in the specific heat might not be visible in a torque measurement. On the other hand, the almost temperature-independent FFLO-transition line would be hardly observable in the specific heat. These arguments further emphasize the gain in information from a combination of the two methods. The different field dependences of the FFLO transition in the two data sets might originate from the metastability that was observed at the lowest temperatures. The most important point is, however, that the magnetic torque sensor was mounted on a highly precise rotor which allowed us to align the sample much more accurately. In the specific-heat experiments it was not possible to align the sample better than $\sim 1^{\circ}$ parallel to the planes. The sensitivity of the FFLO phase on the exact field alignment will be discussed below.

\section{ANISOTROPY OF THE MAGNETIC TORQUE FOR IN-PLANE MAGNETIC FIELDS OF DIFFERENT ORIENTATIONS}

The symmetry of the order parameter of $\kappa$-(BEDT$\mathrm{TTF})_{2} \mathrm{Cu}(\mathrm{NCS})_{2}$ is still a subject of controversy (e.g., the specific-heat results ${ }^{26}$ point to either $s$ - or $d$-wave symmetry). In the Pauli-limiting case (without FFLO state) for $s$-wave superconductivity the upper critical field is expected to be independent of the direction of the magnetic field, as long as $H$ lies in the conducting BEDT-TTF plane. However, this is expected to change in the presence of the FFLO state for $d$-wave superconductors. Maki and $\mathrm{Won}^{27}$ predicted a pronounced in-plane anisotropy of the upper critical field for this case. Our torque measurements represent a good tool to investigate whether such anisotropy exists. During the measurements of the data presented in Sec. III, the field was oriented along the $b$ direction of the sample. For a $d$-wave order parameter an in-plane anisotropy of the upper critical field line should exist. Therefore, we turned the sample by $45^{\circ}$ on the cantilever and repeated the measurements with magnetic 


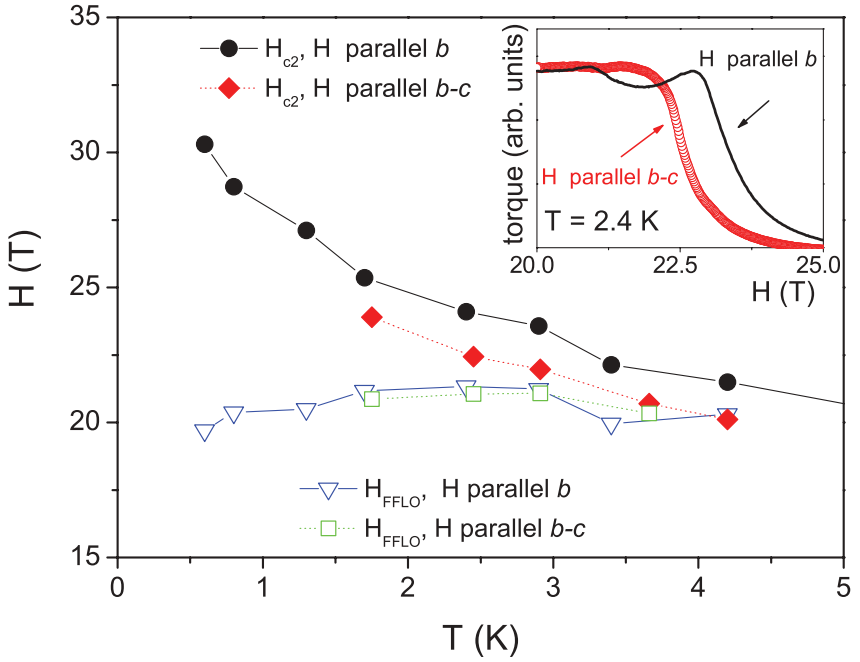

FIG. 3. (Color online) Phase diagram comparing the upper critical field and the FFLO-transition line for in-plane magnetic fields along the $b$ and the $b-c$ directions. Inset: Magnetic torque of $\kappa$-(BEDT-TTF $)_{2} \mathrm{Cu}(\mathrm{NCS})_{2}$ at $2.4 \mathrm{~K}$ for the two in-plane orientations of the applied field.

field along the in-plane $b-c$ direction. The phase diagram obtained is presented in Fig. 3. Compared to the results in the $b$ direction, the upper-critical-field line is reduced by about $1 \mathrm{~T}$ in fields applied along the $b-c$ direction. Considering the fact that the sample had to be aligned for both orientations separately for an in-plane field orientation, this is only a minor difference. The temperature dependence of $H_{\mathrm{c} 2}$ follows more or less that for magnetic field applied along the $b$ direction. $H_{\text {FFLO }}$, which is basically identical to $H_{\mathrm{P}}$, remains the same for both orientations of the sample. The anomaly is less pronounced for the orientation at $45^{\circ}$ but still clearly visible. Although our measurement time in the high-magnetic-field laboratory was not sufficient for a more detailed investigation, we can conclude that for a $d$-wave scenario, the expected $H_{\mathrm{c} 2}$ anisotropy is expected to be much larger than that observed in Fig. 3. A minor anisotropy of the pairing symmetry, however, may be present.

\section{OUT-OF-PLANE ANGULAR DEPENDENCE OF THE MAGNETIC TORQUE}

Turning the field in small steps out of the orientation exactly parallel to the superconducting layers of the sample allows us to induce orbital currents in the form of vortices in a controlled manner. With this experiment we aim at investigating how these currents interact with the FFLO state and how robust the FFLO state is in the presence of orbital currents. This series of experiments was performed at $T=2.0 \mathrm{~K}$ and the sample has been tilted in small steps of $0.2^{\circ}$ out of the parallel orientation (starting from a field orientation along the crystalline $b$ axis). The data are presented in Fig. 4. Between $0^{\circ}$ (field alignment parallel to the superconducting BEDT-TTF layers) and $0.2^{\circ}$ the transition at $H_{\mathrm{c} 2}$ sharpens clearly. (Note that the data taken at $0^{\circ}$ are not identical to the data shown in Fig. 1. The sample orientation needed to be readjusted prior to this experiment.) Turning the sample further out of the parallel alignment leads to a broadening of the $H_{\mathrm{c} 2}$ transition. Besides the pronounced
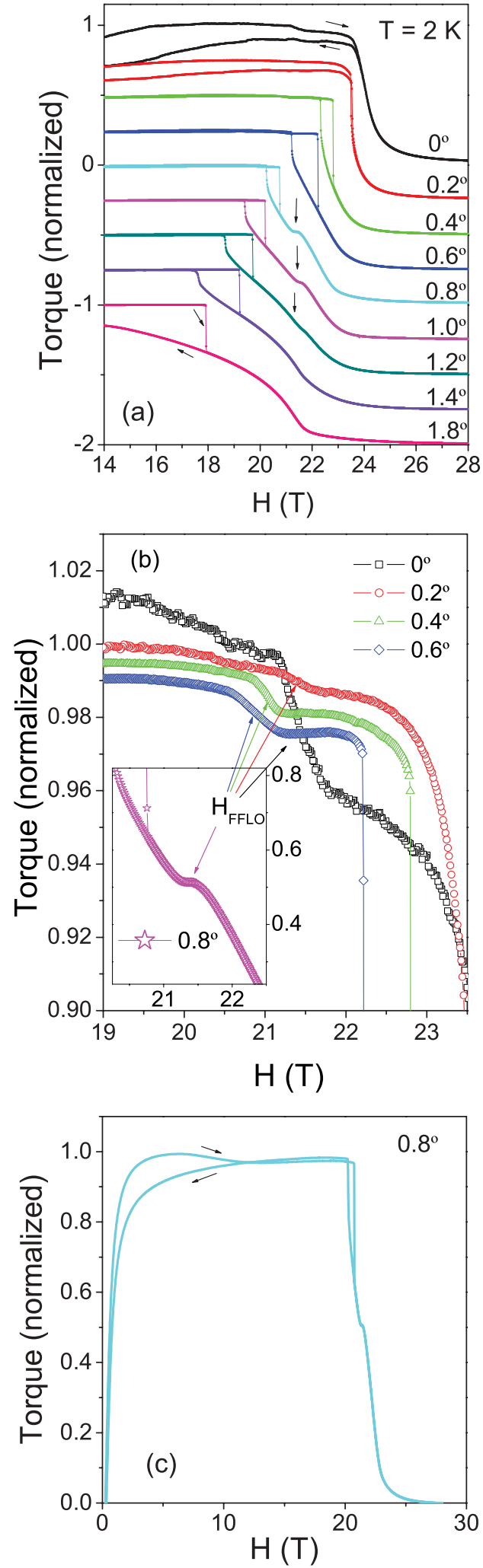

FIG. 4. (Color online) (a) Magnetic torque of $\kappa$-(BEDT$\mathrm{TTF})_{2} \mathrm{Cu}(\mathrm{NCS})_{2}$ for tilted angles between the magnetic field and the superconducting planes between $0^{\circ}$ and $1.8^{\circ}$. (b) Enlargements of the data showing the small anomaly at the FFLO transitions close to $21 \mathrm{~T}$. The curves in (a) are shifted with respect to each other for clarity. (c) Data taken at $0.8^{\circ}$ shown over the entire magnetic-field range. 
$H_{\mathrm{c} 2}$ transition two smaller anomalies are visible: An extremely sharp jump related to a first-order transition occurs close to the lower onset of the broadened $H_{\mathrm{c} 2}$ transition, with a distinct hysteresis in field. The larger the angle, the lower the field where these jumps occur and the more pronounced the hysteresis. Furthermore, zooming in on the upper onset of the $H_{\mathrm{c} 2}$ transition Fig. 4(b) reveals that the small step, which we ascribed to the FFLO transition, remains at $\sim 21 \mathrm{~T}$ and shows hardly any angular dependence. Finally, at $0.8^{\circ}$ the sharp jumps drop below $21 \mathrm{~T}$ on crossing the FFLO transition. At larger angles there are still anomalies occurring at $21 \mathrm{~T}$ (indicated by the vertical arrows in Fig. 4) located on the broadened part of the $H_{\mathrm{c} 2}$ transition at fields above the sharp jumps. For angles larger than $1.4^{\circ}$, the superconductivity finally no longer reaches the Pauli limit and the FFLO transitions disappear.

The sharp jumps represent a significant fraction of the total change in the diamagnetic signal between the superconducting and normal state and it is clear that they need to be considered as part of the $H_{\mathrm{c} 2}$ transition. Finally, at $1.8^{\circ}$, the jump still appears during the upward field sweep but upon lowering the field the transition no longer appears and the curve remains on a metastable lower branch down to $0 \mathrm{~T}$.

The fact that this sharp jump appears only for nonparallel field orientations suggests that this first-order transition is related to vortices which enter the volume of the crystal. It is well known that the superconducting transition of layered organic superconductors, such as $\kappa$-(BEDT) $)_{2} \mathrm{Cu}(\mathrm{NCS})_{2}$, is strongly governed by critical phase fluctuations, similar to the cuprate high-temperature superconductors. In such systems, the superconducting transition appears as a broadened crossover in magnetic fields and the detectable transition is reduced toward a first-order vortex-melting transition at which the global phase coherence of the condensate is formed and zero resistivity occurs. ${ }^{28}$ Therefore, the sharp jumps are most probably related to vortex melting. The hysteresis is very pronounced. Finally, in the measurement obtained at $1.8^{\circ}$, the transition is completely "undercooled" toward a vortex-glass transition. ${ }^{29,30}$ The vortex density is increasing rapidly with increasing angle which makes flux pinning effects and vortex-vortex interactions more effective. The time scale imposed by our magnetic-field sweep rate becomes too short for vortices to relax into the crystalline ground state at this angle.

One detail of the FFLO transition at tilted angles is remarkable: The data obtained at small angles below $0.6^{\circ}$ show that the FFLO transition appears within the phase-coherent vortex-solid phase, evidenced by a downward step, which is related to the spatial modulation of the order parameter reducing the superfluid density. However, for angles higher than $0.6^{\circ}$, when the FFLO transition occurs within the phaseincoherent vortex-liquid phase, the FFLO transition is reflected by a more pronounced upward step at slightly higher magnetic field, which means that the superconductivity is strengthened by the establishment of the FFLO state. The fact that these features always remain at the Pauli limit at about $21 \mathrm{~T}$ indicates that they indeed are related to the formation of the FFLO state. The FFLO transition is observed here to appear in two different superconducting states: within the phase-coherent Abrikosov

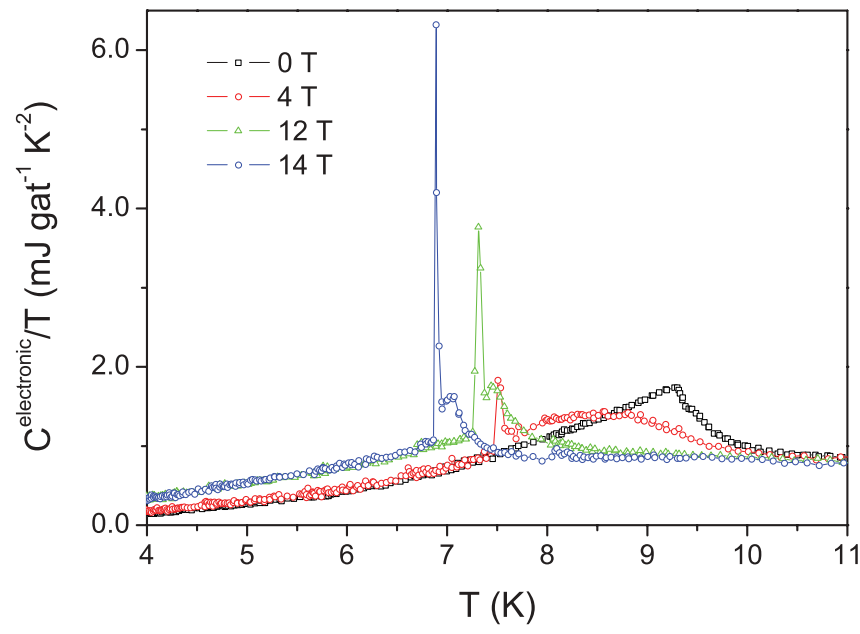

FIG. 5. (Color online) Specific heat of $\kappa$-(BEDT$\mathrm{TTF})_{2} \mathrm{Cu}(\mathrm{NCS})_{2}$ in magnetic fields tilted by about $3^{\circ}$ away from parallel in-plane alignment.

state and within the phase-incoherent vortex-liquid state. The latter is dominated by strong fluctuations in the phase of the order parameter. In the phase-incoherent liquid-vortex state the orbital currents are most likely weakened by the loss of phase coherence. Orbital currents then become dissipative and therefore decay rapidly. The spatial FFLO modulation of the order parameter within the vortex-liquid state, therefore, most likely restores a part of the phase coherence by confining the vortices in the regions of small amplitude of the order parameter.

To further investigate the thermodynamic origin of the vortex transition, the specific heat of a BEDT-TTF $)_{2} \mathrm{Cu}(\mathrm{NCS})_{2}$ sample was measured with a microrelaxation calorimeter with the magnetic field oriented about $3^{\circ}$ away from the parallel orientation of the superconducting planes. The results are presented in Fig. 5. In accordance with the torque experiments, small first-order spikelike anomalies are observed in the specific heat for these tilted field orientations of the sample. This behavior confirms the thermodynamic nature of the additional vortex-melting phase transitions observed in the torque experiments.

This first-order anomaly in the data shown in Fig. 5 is very similar to the first-order vortex-melting transition observed in the specific heat of $\mathrm{YBa}_{2} \mathrm{Cu}_{3} \mathrm{O}_{7},{ }^{31} \mathrm{NdBa}_{2} \mathrm{Cu}_{3} \mathrm{O}_{7},{ }^{32}$ and recently also in $\mathrm{Nb}_{3} \mathrm{Sn}^{33}$ The upward step indicates an increase in the number of degrees of freedom, typical for a solid-to-liquid melting transition. In magnetic fields applied perpendicular to the layers the vortexmelting transition vanishes in the specific heat, simply because of the small number of vortices at low magnetic fields, which make the transition anomaly too small to be detected. $^{20}$

The data sets measured at different orientations of the superconducting layers with respect to the magnetic field demonstrate the effect of introducing vortices into a superconductor. The most surprising result is that the formation of the FFLO state is rather robust in the presence of vortices. Obviously, the phase can be formed out of the liquid-vortex phase above the vortex-melting transition, which is expected 
to be strongly governed by phase fluctuations. The FFLO state persists as long as superconductivity reaches the Pauli limit.

\section{CONCLUSION}

For $\kappa$-(BEDT-TTF $)_{2} \mathrm{Cu}(\mathrm{NCS})_{2}$, the phase diagram obtained from magnetic-torque measurements strongly corroborates the presence of a novel superconducting high-field state in magnetic fields beyond $21 \mathrm{~T}$ applied parallel to the superconducting layers. The transition between the conventional superconducting low-field phase and the high-field state is of thermodynamic nature and arises always close to the Pauli limit of about $21 \mathrm{~T}$. In addition, the upper-critical-field line shows a distinct increase at low temperatures. Numerous theoretical approaches are available in the literature that describe possible phase diagrams of a superconductor with an FFLO state. ${ }^{34-36}$ For superconductors in the ultraclean limit, such as $\kappa$-(BEDT-TTF $)_{2} \mathrm{Cu}(\mathrm{NCS})_{2}$, the FFLO state was calculated to occur below a crossover temperature $T_{0}$. For $\kappa$-(BEDT-TTF $)_{2} \mathrm{Cu}(\mathrm{NCS})_{2}$ we find $T_{0} \approx 4 \mathrm{~K}$ as onset temperature of the additional phase transition in our experiment. The upturn of $H_{\mathrm{c} 2}$ below $T_{0}$ is in accordance with the expected behavior for the FFLO state. ${ }^{20}$ The good agreement with theoretical predictions leads us to conclude that the additional phase transition within the superconducting state signals the occurrence of an FFLO state at high magnetic fields.

Our experiments show some minor in-plane anisotropy of the upper critical field at low temperatures once the magnetic field is aligned into different in-plane directions. However, this anisotropy is much smaller than expected for a $d$-wave superconductor. Furthermore, the FFLO transition remains robust when the magnetic field is tilted out of the superconducting planes, until at angles larger than about $1.2^{\circ}$ the superconducting state does not reach the Pauli limit anymore. The presence of vortices at tilted angles manifests itself in the form of another very sharp first-order thermodynamic transition which shows all the characteristics of a vortex-melting transition. It would be interesting to investigate the microscopic realization of the vortex matter in the presence of the spatial modulation of the order parameter. Theory predicts rather complicated vortex structures for this case. $^{37}$

\section{ACKNOWLEDGMENT}

Part of this work has been supported by EuroMagNET under the EU Contract No. 228043.
*Corresponding author; lortz@ust.hk

${ }^{1}$ L. P. Gor'kov, Sov. Phys. JETP 10, 593 (1960).

${ }^{2}$ A. K. Clogston, Phys. Rev. Lett. 9, 266 (1962).

${ }^{3}$ B. S. Chandrasekhar, Appl. Phys. Lett. 1, 7 (1962).

${ }^{4}$ P. Fulde and R. A. Ferrell, Phys. Rev. 135, A550 (1964).

${ }^{5}$ A. I. Larkin and Y. N. Ovchinnikov, Sov. Phys. JETP 20, 762 (1965).

${ }^{6}$ A. Bianchi, R. Movshovich, C. Capan, P. G. Pagliuso, and J. L. Sarrao, Phys. Rev. Lett. 91, 187004 (2003).

${ }^{7}$ H. A. Radovan, N. A. Fortune, T. P. Murphy, S. T. Hannahs, E. C. Palm, S. W. Tozer, and D. Hall, Nature (London) 425, 51 (2003).

${ }^{8}$ M. Kenzelmann, S. Gerber, N. Egetenmeyer, J. L. Gavilano, Th. Strässle, A. D. Bianchi, E. Ressouche, R. Movshovich, E. D. Bauer, J. L. Sarrao, and J. D. Thompson, Phys. Rev. Lett. 104, 127001 (2010); G. Koutroulakis, M. D. Stewart, Jr., V. F. Mitrovic, M. Horvatic, C. Berthier, G. Lapertot, and J. Flouquet, ibid. 104, 087001 (2010).

${ }^{9}$ M. Kenzelmann, Th. Strässle, C. Niedermayer, M. Sigrist, B. Padmanabhan, M. Zolliker, A. D. Bianchi, R. Movshovich, E. D. Bauer, J. L. Sarrao, and J. D. Thompson, Science 321, 1652 (2008).

${ }^{10}$ R. Casalbuoni and G. Nardulli, Rev. Mod. Phys. 76, 263 (2004).

${ }^{11}$ Y. Matsuda and H. Shimahara, J. Phys. Soc. Jpn. 76, 051005 (2007).

${ }^{12}$ H. Shimahara, Phys. Rev. B 50, 12760 (1994).

${ }^{13}$ H. Shimahara, J. Phys. Soc. Jpn. 66, 541 (1997).

${ }^{14}$ H. Burkhardt and D. Rainer, Ann. Phys. (Berlin) 506, 181 (1994).

${ }^{15}$ J. Singleton, J. A. Symington, M.-S. Nam, A. Ardavan, M. Kurmoo and P. Day, J. Phys. Condens. Matter 12, L641 (2000).

${ }^{16}$ M. A. Tanatar, T. Ishiguro, H. Tanaka, and H. Kobayashi, Phys. Rev. B 66, 134503 (2002).

${ }^{17}$ S. Uji, T. Terashima, M. Nishimura, Y. Takahide, T. Konoike, K. Enomoto, H. Cui, H. Kobayashi, A. Kobayashi, H. Tanaka, M. Tokumoto, E. S. Choi, T. Tokumoto, D. Graf, and J. S. Brooks, Phys. Rev. Lett. 97, 157001 (2006).
${ }^{18}$ I. J. Lee, M. J. Naughton, G. M. Danner, and P. M. Chaikin, Phys. Rev. Lett. 78, 3555 (1997).

${ }^{19}$ J. Shinagawa, Y. Kurosaki, F. Zhang, C. Parker, S. E. Brown, D. Jérome, K. Bechgaard, and J. B. Christensen, Phys. Rev. Lett. 98, 147002 (2007).

${ }^{20}$ R. Lortz, Y. Wang, A. Demuer, P. H. M. Böttger, B. Bergk, G. Zwicknagl, Y. Nakazawa, and J. Wosnitza, Phys. Rev. Lett. 99, 187002 (2007).

${ }^{21}$ B. Bergk, A. Demuer, I. Sheikin, Y. Wang, J. Wosnitza, Y. Nakazawa, R. Lortz, Physica C 470, 586 (2010).

${ }^{22}$ K. Cho, B. E. Smith, W. A. Coniglio, L. E. Winter, C. C. Agosta, and J. A. Schlueter, Phys. Rev. B 79, 220507 (2009).

${ }^{23}$ H. Urayama, H. Yamochi, K. Nozawa, T. Sugano, M. Kinoshita, S. Sato, K. Oshima, A. Kawamoto, and J. Tanaka, Chem. Lett. 17, 55 (1988).

${ }^{24}$ J. Wosnitza, Fermi Surfaces of Low-Dimensional Organic Metals and Superconductors (Springer, Berlin, 1996).

${ }^{25}$ L. W. Gruenberg and L. Gunther, Phys. Rev. Lett. 16, 996 (1966).

${ }^{26}$ J. Müller, M. Lang, R. Helfrich, F. Steglich, and T. Sasaki, Phys. Rev. B 65, 140509 (2002); O. J. Taylor, A. Carrington, and J. A. Schlueter, Phys. Rev. Lett. 99, 057001 (2007).

${ }^{27}$ K. Maki and H. Won, Czech. J. Phys. 46, 1035 (1996).

${ }^{28}$ W. K. Kwok, J. Fendrich, S. Fleshler, U. Welp, J. Downey, and G. W. Crabtree, Phys. Rev. Lett. 72, 1092 (1994).

${ }^{29}$ D. S. Fisher, M. P. A. Fisher, and D. A. Huse, Phys. Rev. B 43, 130 (1991).

${ }^{30}$ R. Lortz, C. Meingast, A. I. Rykov, and S. Tajima, J. Low Temp. Phys. 147, 365 (2007).

${ }^{31}$ A. Schilling, R. A. Fisher, N. E. Phillips, U. Welp, W. K. Kwok, and G. W. Crabtree, Phys. Rev. Lett. 78, 4833 (1997).

${ }^{32}$ T. Plackowski, Y. Wang, R. Lortz, A. Junod, and T. Wolf, J. Phys. Condens. Matter 17, 6871 (2005). 
${ }^{33}$ R. Lortz, F. Lin, N. Musolino, Y. Wang, A. Junod, B. Rosenstein, and N. Toyota, Phys. Rev. B 74, 104502 (2006).

${ }^{34}$ D. F. Agterberg and K. Yang, J. Phys. Condens. Matter 13, 9259 (2001)

${ }^{35}$ M. Houzet and A. Buzdin, Phys. Rev. B 63, 184521 (2001).
${ }^{36}$ M. Tachiki, S. Takahashi, P. Gegenwart, M. Weiden, M. Lang, C. Geibel, F. Steglich, R. Modler, C. Paulsen, and Y. Onuki, Z. Phys. B 100, 369 (1996).

${ }^{37}$ K. Yang and A. H. MacDonald, Phys. Rev. B 70, 094512 (2004). 